\title{
Wege zur dauerhaften Integration
}

\section{Dem Umweltcontrolling steht heute eine Vielzahl an Instrumenten zur Auswahl. Ein Unternehmen muss in der Erwartung der Nutzenpotenziale entscheiden, welches Instrument mit welcher IT-Lösung einmalig angewendet beziehungs- weise langfristig in der Organisation institutionalisiert wird. Das entwickelte Implementierungs- und Institutionalisierungskonzept hilft den Unternehmen bei diesen Entscheidungsprozessen.}

$\mathrm{U}$ Von Michael Steinfeldt nternehmen begegnen drei Fragestellungen wenn sie die interne Informationsversorgung für eine umweltorientierte Unternehmensfïhrung optimieren wollen. Zu berïcksichtigen sind hier die Eignung der verschiedenen Umweltcontrolling(UC)-Instrumente, die Unterstützung der Instrumente durch Informationstechnologien (IT) und die organisatorische Umsetzung der Einführung und der dauerhaften Anwendung der Instrumente.

Für die Bewältigung dieser drei Herausforderungen wurden im Forschungsprojekt INTUS entsprechende Konzepte für Unternehmen entwickelt (1).

\section{- Herausforderung organisato- rische Integration}

In Konkurrenz zu dauerhaften Anwendungen von Instrumenten des Umweltcontrolling stehen Analysetools zur einmaligen oder unregelmäßigen Nutzung, die nicht in die Unternehmensorganisation und deren Abläufe eingebunden sind. Daher stellt sich aus organisatorischer Sicht zunächst die Frage, ob der erwartete Langzeitnutzen aus der dauerhaften Anwendung und der organisatorischen Verankerung der UC-Instrumente einerseits den Aufwand für die Implementierung und Aufrechterhaltung rechtfertigt, und, ob dieser andererseits höher ist als der Nutzen einmaliger Analysen.

Für den erfolgreichen Einsatz eines UC-Instrumentes im Unternehmen ist neben der absoluten Höhe des Nutzens der Implementierungsaufwand sowie der spätere laufende Aufwand zur Aufrechterhaltung der Funktionalität der Instrumentenlösung ausschlaggebend. Mit dem Ziel, diesen Aufwand zu reduzieren, wurde ein Phasenkonzept zur Implementierung und Institutionalisierung von Instrumenten des UC ent- wickelt. Diesen Prozess als organisatorische Innovation aufzufassen, machte es möglich, die vielfältigen Erkenntnisse der betrieblichen Innovationsforschung zu nutzen. In der Reflexion zahlreicher Phasen- und Promotorenmodelle für betriebliche Innovationsprozesse umfasst das entwickelte Phasenkonzept in Anlehnung an Tolbert/Zucker vier Phasen: Ideengenerierung, spezifische Problemlösung, Implementierung und Alltagsanwendung (2). Durch die vier Phasen wird der Gesamtprozess einerseits genügend strukturiert, andererseits ist Platz für weitere Detaillierungen zur Ausgestaltung der einzelnen Phasen des Innovationsprozesses. Neben der Ausfïhrungsqualität, die durch methodische Professionalität und Systematik bestimmt wird, lassen sich für den Implementierungs- und Institutionalisierungsprozess drei weitere wichtige Einflussgrößen ableiten, die im Projekt vertieft bearbeitet wurden:

- Instrumenten-Know-How,

- IT-Unterstïtzung und Integration sowie die - organisationale Lernfähigkeit des Unternehmens.
Bezïglich der erstgenannten Einflussgröße wurde im Projekt untersucht, in wie weit die Instrumente Umweltbilanz, Umweltkennzahlen und Flusskostenrechnung zur Unterstïtzung der Aufgaben des Umweltmanagements geeignet und kombinierbar sind. Hierzu wurde ein Konzept zur Entscheidungsfindung über den Einsatz von betrieblichen Umweltbilanzen, Umweltkennzahlen und Flusskostenrechnung erarbeitet (3). Das erarbeitete IT-Konzept zeigt, welche Lösungen grundsätzlich für die Bereitstellung der UC-Instrumente bestehen und welche Lösungen entsprechend dem heutigen Entwicklungsstand empfohlen werden können. In Bezug auf die dritte Einflussgröße wurden Ansätze des organisationalen Lernens sowie Promotorenmodelle für Innovationsprozesse analysiert und für diesen Anwendungskontext angepasst. Hierbei galt es beispielsweise zu klären, wie die im zumeist interdisziplinären Projektteam vertretenden Fachleute aktiv in die einzelnen Arbeits- und Lernprozesse einzubeziehen sind.

Außerdem konnte im Forschungsprojekt festgestellt werden, dass sich die Bedeutung der Einflussgrößen im Projektverlauf ändert (siehe Abb. 1). Während das Instrumenten-Thema, damit ist insbesondere die Entwicklung der unternehmensspezifischen UC-Instrumentenlösung gemeint, besonders zu Beginn eine große Bedeutung besitzt, gewinnen die anderen beiden Einflussgrößen im Projektverlauf erst später an Bedeutung. Für das Thema IT-Unterstützung / Integration kann festgestellt werden, dass es in den Phasen der spezifischen Problemlösung und der Implementierung eine große Rolle spielt, wohingegen das Thema der organisatorischen Lernfähigkeit in der Objektivierungs-

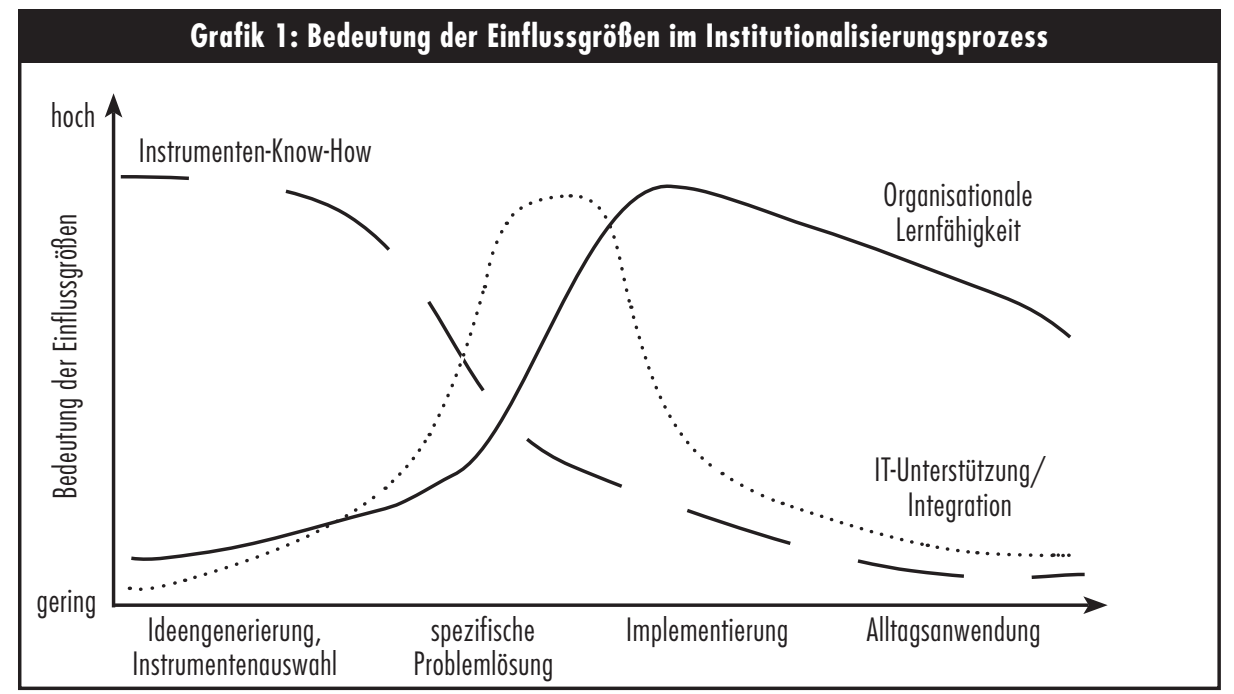

Quelle: INTUS vgl. Anmerkungen 

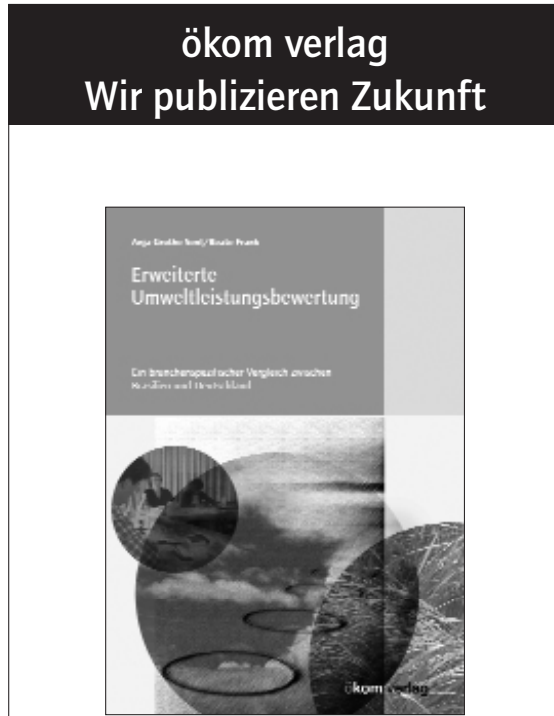

Anja Grothe-Senf, Beate Frank

Erweiterte Umweltleistungsbewertung

\section{Ein branchenspezifischer Vergleich zwischen Brasilien und Deutschland}

Mit Unternehmen der Pharma-, Chemie- und Textilbranche aus Deutschland und Brasilien führten die Autorinnen eine „erweiterte Umweltleistungsbewertung" durch. Sie gibt Auskunft darüber, welche Rolle das Ziel der Nachhaltigkeit innerhalb der Unternehmensziele hat. Das Buch stellt Bewertungsmodell und Ergebnisse vor und identifiziert die Haupteinflussfaktoren. Mit der beiliegenden CD-Rom können die Leser die er-weiterte Umweltleistungsbewertung im eigenen Unternehmen durchführen: Wie weit sind Sie auf dem Weg zur Nachhaltigkeit?

Anja Grothe-Senf, Beate Frank ökom verlag, München 2003 215 Seiten incl. CD

27,- € (zzgl. Versandkosten) ISBN 3-936581-24-X

Erhältlich bei CONSODATA ONE-TO-ONE Semmelweisstraße 8, D-82152 Planegg Fon $++49 /(0) 89 / 857$ 09-155 Fax $++49 /(0) 89 / 857$ 09-131 kontakt@oekom.de phase mit der betrieblichen Umsetzung in die Aufbau- und Ablauforganisation seinen Höhepunkt hat.

Die Prioritätenverschiebungen im Prozessverlauf werden im entwickelten Vorgehenskonzept dadurch berïcksichtigt, dass den einzelnen Prozessphasen relevante Leitfragen zugeordnet und die themenspezifisch erarbeiteten Konzepte entsprechend eingebunden sind (4).

Für die optimale Prozessgestaltung der Implementierung und Institutionalisierung von Instrumenten des UC erweist es sich als sehr sinnvoll, die verschiedenen Dimensionen des organisationalen Lernens in den Prozessphasen unterschiedlich stark zu berücksichtigen. In den ersten beiden Phasen Ideengenerierung/Instrumentenauswahl und spezifische Problemlösung handelt es sich in erster Linie um individuelles und projektgruppenspezifisches Lernen, wohingegen die Phasen Implementierung und Alltagsanwendungen stark durch Organisationslernen geprägt sind. Entsprechend sind in den einzelnen Prozessphasen unterschiedliche Methoden des organisationalen Lernens (z.B. Brainstorming, -writing, Morphologischer Kasten, Aktionslernen, Projekt-Prototyping, Organisationsgesundheitsbild) anzuwenden.

Außerdem sollte der Nutzen der innovativen UCInstrumentenlösung den erforderlichen Aufwand zur Implementierung und Institutionalisierung rechtfertigen. Das im Projekt entwickelte Phasenkonzept sieht daher bewusst entscheidungsorientierte Abbruchmöglichkeiten für den Fall vor, dass der zu erwartende Nutzen des Einsatzes von UC-Instrumenten eine dauerhafte Einführung und organisatorische Verankerung nicht rechtfertigt.

Des weiteren wurde untersucht, welche umweltbezogenen organisationalen Lernprozesse in Verbindung mit dem Einsatz von UC-Instrumenten erwartbar sind (9). Es zeigt sich, dass die UC-Instrumente primär Single-Loop-Lernprozesse, also Anpassungslernen auslösen. Das heißt, es wird ein auf Effizienz zielendes Anpassungslernen vollzogen und bestehende Problemlösungen optimiert. Darüber hinaus unterstützt der institutionalisierte Einsatz der Instrumente die Dynamisierung des kontinuierlichen Verbesserungsprozesses und regt $\mathrm{zu}$, ,Quantensprüngen“ an, so dass sich die Wahrscheinlichkeit für Double-Loop-Lernen oder Veränderungslernen erhöht, bei dem organisationale Verhaltensmuster, Normen etc. kritisch reflektiert und geändert werden.

\section{Fazit}

Das entwickelte Phasenkonzept ermöglicht es Unternehmen, in optimierten Lernprozessen Instrumente des UC in die Unternehmensorganisation zu implementieren und institutionalisieren und bindet dabei die im Forschungsprojekt entwickelten Konzepte zur Entscheidungsfindung des Instrumenteneinsatzes sowie zur IT- Unterstiitzung ein. Die Reflexion der theoretischen Konzepte unter anderem zu betrieblichen Innovationsprozessen und zu organisationalem Lernen hat geholfen, dieses schlüssige Phasenkonzept zu entwickeln, wenngleich einschränkend festzustellen ist, dass die Theorie weniger beitragen konnte als ursprünglich erhofft.

Die grundsätzliche Frage nach dem AufwandNutzen-Verhältnis der dauerhaften Integration der jeweiligen Instrumente ist immer noch entscheidend für die Priorität entsprechender Umweltprojekte. Wenn der Nettonutzen als zu gering eingeschätzt wird oder gar tatsächlich zu gering ist, werden die Projekte entweder nicht durchgeführt, oder sie bekommen eine niedrige Priorität im Unternehmen, was die Umsetzung deutlich erschwert.

\section{Anmerkungen}

(1) Für Informationen zu INTUS vgl. Spath, D./ Lang, C./ Loew, T.: Broschüre Umweltcontrolling in produzierenden Unternehmen. Stuttgart 2003. Publikationen unter www. bum.iao.fraunhofer.de/downloads und www.ioew.de (2) Tolbert, P./ Zucker, L.: The Institutionalisation of Institutional Theory. In Clegg, S./ Hardy, C./ Nord, W. (Hg.): Handbook of Organization Studies. London 1996,

S. $175-190$.

(3) Loew, T.: Konzept zur Entscheidungsfindung über den Einsatz von betrieblichen Umweltbilanzen, Umweltkennzahlen und Flusskostenrechnung. Berlin 2003.

(4) Steinfeldt, M./ Lang, C.: Konzept zur Implementierung und Institutionalisierung von Instrumenten des Umweltcontrolling. Berlin, Stuttgart 2004.

(5) Steinfeldt, M./ Hoffmann, E.: Organisationales Lernen und umweltbezogene Lernprozes se. Schriftenreihe des IÖW 170/03, Berlin 2003.

\section{Der Autor}

Michael Steinfeldt ist wissenschaftlicher Mitarbeiter im Forschungsfeld Ökologische Unternehmenspolitik am Institut für ökologische Wirtschaftsforschung (IÖW).

Kontakt: IÖW, Potsdamer Str. 105, 10785 Berlin. Tel. 030-8845940,

E-Mail: Michael.Steinfeldt@ioew.de 
(c) 20I0 Authors; licensee IÖW and oekom verlag. This is an article distributed under the terms of the Creative Commons Attribution Non-Commercial No Derivates License (http://creativecommons.org/licenses/by-nc-nd/3.o/), which permits unrestricted use, distribution, and reproduction in any medium, provided the original work is properly cited. 